\title{
Fluctuation Forces and Wetting Layers in Colloid-Polymer Mixtures
}

\author{
Y. Hennequin, ${ }^{1, *}$ D. G. A. L. Aarts, ${ }^{2}$ J. O. Indekeu, ${ }^{3}$ H. N. W. Lekkerkerker, ${ }^{4}$ and D. Bonn ${ }^{1,5}$ \\ ${ }^{1}$ Van der Waals-Zeeman Institute, University of Amsterdam, Valckenierstraat 65, 1018XE Amsterdam, The Netherlands \\ ${ }^{2}$ Physical and Theoretical Chemistry Laboratory, University of Oxford, South Parks Road, Oxford OX1 3QZ, United Kingdom \\ ${ }^{3}$ Instituut voor Theoretische Fysica, Katholieke Universiteit Leuven, Celestijnenlaan 200D, B-3001 Leuven, Belgium \\ ${ }^{4}$ Van 't Hoff Laboratory, Utrecht University, Padualaan 8, 3584CH Utrecht, The Netherlands \\ ${ }^{5}$ Laboratoire de Physique Statistique, Ecole Normale Supérieure, 24 Rue Lhomond, 75231 Paris cedex 05, France
}

(Received 20 May 2007; published 2 May 2008)

\begin{abstract}
We present confocal microscopy experiments on the wetting of phase-separated colloid-polymer mixtures. We observe that an unusually thick wetting layer of the colloid-rich phase forms at the walls of the glass container that holds the mixture. Because of the ultralow interfacial tension between the colloid-rich and the polymer-rich phases, the thermally activated roughness of the interfaces becomes very big and measurable. We observe that close to the critical point the roughness of the interface between the wetting layer and the polymer-rich phase decreases with decreasing layer thickness: large excursions of the interface are confined in the wetting layer. The measured relationship between the roughness and the thickness of the wetting layer is in qualitative agreement with the predictions of renormalization group theory for short-range forces and complete wetting.
\end{abstract}

DOI: 10.1103/PhysRevLett.100.178305

Wetting layers are ubiquitous in both nature and industry $[1,2]$ and occur in a wide range of systems. For two coexisting phases, Cahn first proposed an argument that explains the formation of a wetting layer of one of the two phases onto a substrate (complete wetting) when approaching the critical point [3]. One classical example is a demixed (two-phase) binary liquid mixture in which the dense phase intrudes between the lighter fluid phase and the vapor. However, once the wetting conditions are set, the remarkable density inversion can only be maintained if forces act between the two film interfaces. For ordinary liquids, these forces are generally van der Waals forces $[1,2]$ which, given their range, invariably lead to wetting layer thicknesses of the order of $100 \AA$.

Liquid interfaces are also rough - they are locally fluctuating due to thermally excited capillary waves [4]. Consequently, the confinement of interface fluctuations in a wetting layer can in principle create an entropic interaction that enters the force balance between surface forces and gravity [5]. The existence of such an entropic force was first established by Helfrich [6] in the case of tensionless membranes. For liquid interfaces, where surface tension dominates, the situation is more complex. From a theoretical point of view, the influence of capillary fluctuations on wetting phenomena has attracted much attention as it could lead to nonuniversal wetting behavior with critical exponents depending explicitly on temperature $[7,8]$. Experimentally, the observation of this entropic repulsion in typical liquid layers is challenging, but some observations have shown that a correct description of adsorption isotherms of molecularly thin films could be achieved if one takes the entropic interaction into account [9-12].

Similarly to simple liquids, mixtures of colloidal particles with nonadsorbing polymer can phase separate into a
PACS numbers: 82.70.Dd, 68.08.Bc, 68.35.Ct, 68.37.- $-\mathrm{d}$

dense colloidal fluid phase (colloidal liquid) and a dilute one (colloidal gas) [13]. This phase separation is due to the depletion attraction that the polymer mediates between the colloids [14-16]. Recently, it has been demonstrated that the depletion attraction between colloids and a hard wall can also lead to interesting wetting phenomena [17-19]. Experiments have shown that a wetting layer of the dense colloidal phase is usually present at the wall of the container $[20,21]$. The exceptional feature of phase-separated colloid-polymer mixtures is the ultralow interfacial tension between the liquid and gas phases [22-24]: it can be a million times smaller than for a typical oil-water interface, thus making the thermal roughness of the interface a thousand times bigger. Consequently, direct observation - in real space and time - of the thermally activated capillary waves becomes possible using optical microscopy [25]. In this Letter, we study the characteristics of wetting layers in phase-separated colloid-polymer mixtures and try to identify the contribution of the micronsized interface fluctuations to the wetting film stability.

Fluorescently labeled poly(methyl methacrylate) colloids of radius $a=68 \mathrm{~nm}$ and nonadsorbing polystyrene polymers of radius of gyration $R_{g}=71 \mathrm{~nm}$ are dispersed in a mixture of cycloheptyl bromide and decalin. The phase behavior is such that the system is homogeneous at low volume fractions of the colloids and the polymers, but separates in two fluid phases (gas-liquid) at intermediate volume fractions, see Fig. 1. This phase separation ends in a bulk critical point.

In order to observe the wetting layers on the vertical cell walls we place a glass container with the mixture in a confocal microscope tilted by $90^{\circ}$ and equipped with high magnification $(\times 100)$ and high-numerical-aperture (1.3) objectives. We perform the measurements for the 


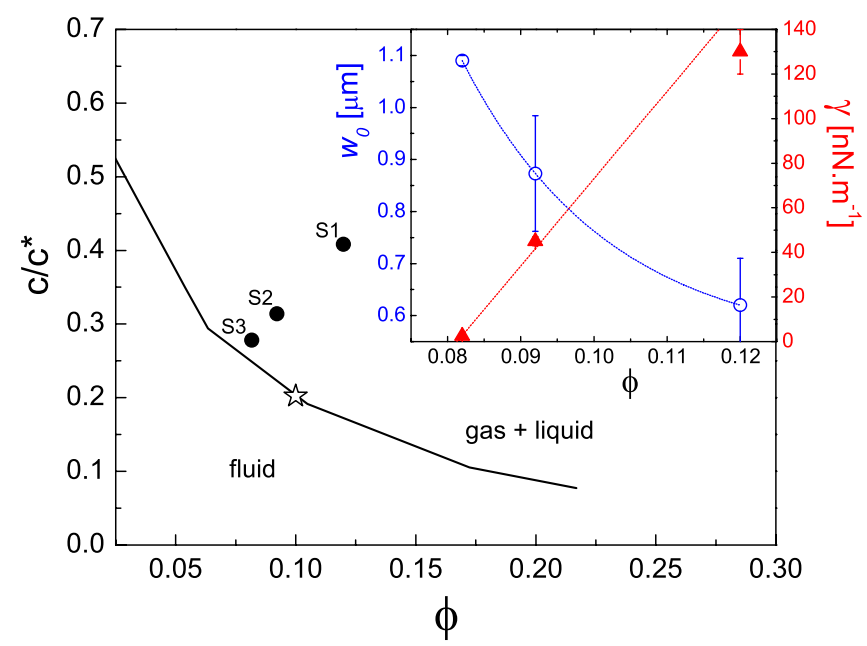

FIG. 1 (color online). Phase diagram of the colloid-polymer system with size ratio $q=R_{g} / a=1.04$ represented as a function of the colloid volume fraction $\phi$ and the polymer concentration $c$ relative to the overlap concentration $c^{*}$. The solid line is the experimental phase boundary between the stable one-fluid and the two-fluid regions and the star marks the approximate location of the critical point. The state points studied are indicated as $\mathrm{S} 1\left(\phi=0.120, c / c^{*}=0.408\right), \mathrm{S} 2(0.092,0.314)$ and S3 (0.082, 0.278). Inset: Averaged roughness $w_{0}$ (circles) and surface tension $\gamma$ (triangles) of the free interface as a function of colloid volume fraction. Lines are guides to the eye.

three different state points indicated in Fig. 1. Figure 2 shows a representative scan through a sample after a few days of equilibration. Successive $x y$ frames $(92 \times$ $92 \mu \mathrm{m}^{2}$ ) spaced by 0.1 or $0.2 \mu \mathrm{m}$ along the $z$ direction (optical axis) are recorded at a given height $h$ above the free interface-gravity points downward the $y$ direction. We first characterize the free interface and measure surface tension, density difference between the two phases and interfacial width. The surface tension $\gamma$ and density difference $\Delta \rho$ are found from the height-height correlation of the capillary fluctuations as described in Ref. [25]. The interfacial width $w$ is deduced by fitting averaged fluorescence intensity profiles along the vertical axis with

$$
\bar{I}(y)=\frac{I_{L}-I_{G}}{2} \operatorname{erf}\left(\frac{y-y_{0}}{w \sqrt{2}}\right)+\frac{I_{L}+I_{G}}{2}
$$

with $y_{0}$ the average position of that interface, and with $I_{L}$ and $I_{G}$ the average fluorescence intensities of the liquid and gas phases, respectively. We find surface tensions ranging from 130 (sample S1), 45 (sample S2) to $2.5 \mathrm{nN} / \mathrm{m}$ (sample S3) and with density differences of approximately 40 , 27 , and $4 \mathrm{~kg} / \mathrm{m}^{3}$, respectively. The width of the free interface $w_{0}$ is typically of the order of $1 \mu \mathrm{m}$, see inset of Fig. 1.

As shown in Fig. 2, we are able to see unambiguously whether a highly fluorescent wetting layer of the colloidrich phase intrudes between the polymer-rich phase and the wall, and we observe such layer for all the state points

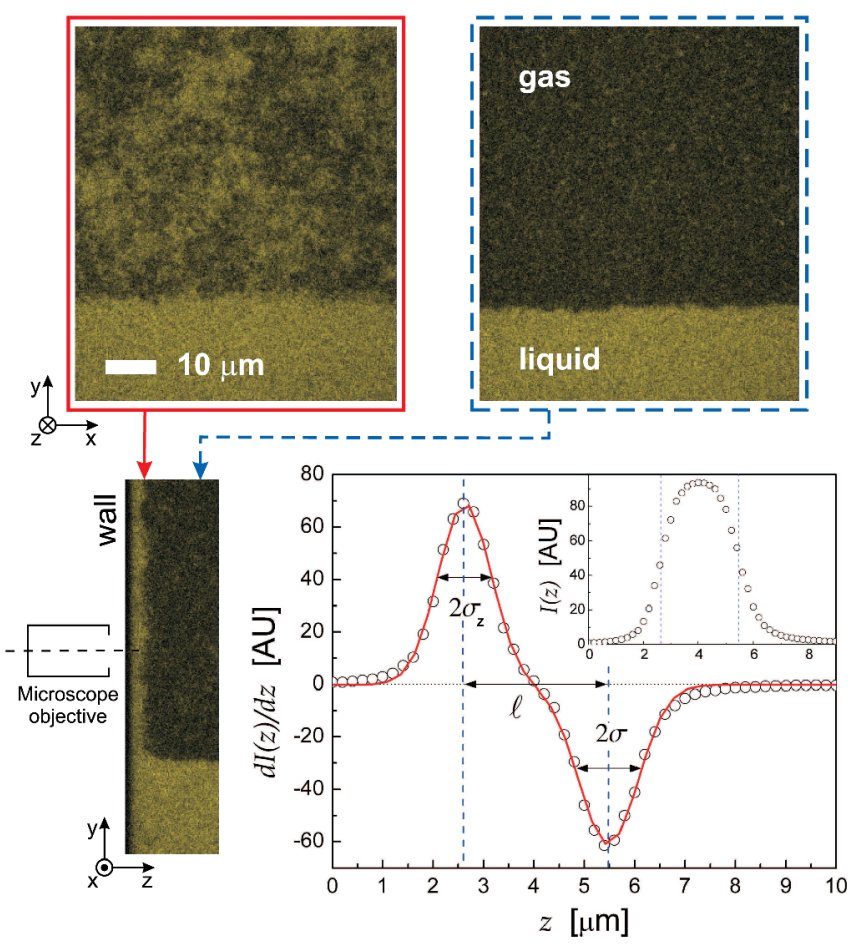

FIG. 2 (color online). Example of a confocal image stack taken at the cell wall and showing the free interface and the vertical wetting layer. Top left: frame taken at the layer's interface. Top right: frame taken in the bulk of the sample. Bottom left: side view of the stack showing the liquid meniscus and the wetting layer at the cell wall. Bottom right: example of the derivative of the frame-averaged fluorescence intensity (inset) as a function of depth $z$; the solid line is a fit that allows to identify (i) the position of the wall and the interface (vertical dotted lines) (ii) the width at the cell wall $\sigma_{z}$ and the width at the interface $\sigma$.

measured. Where possible we follow the layer thickness with time from the phase separation and assume equilibrium once it does not change significantly anymore; this may take several days as opposed to bulk phase separation which takes a few hours to complete. Once established the layer usually persists over the time of the experiment which could be up to one month.

To characterize the wetting layers quantitatively, we measure the fluorescence intensity along the optical axis at different heights above the free interface, see Fig. 2(bottom). Each intensity profile is a convolution between the density profile of the colloids and the instrument resolution (Gaussian with $\sigma_{z} \simeq 0.5 \mu \mathrm{m}$ ). If we assume that the interface fluctuations are normally distributed around the mean interface position $z_{2}$, the density profile can also be described by Eq. (1) $-w$ is now the width of the interface between wetting layer and bulk gas - but cut off (step function) at the cell wall $z_{1}$. We obtain the mean layer thickness $\ell=z_{2}-z_{1}$ and calculate the width of the wetting layer interface $w$ using the convolution approximation

$$
\sigma^{2}=\sigma_{z}^{2}+w^{2}
$$


where $\sigma$ is the apparent width of the wetting layer interface.

Figure 3(a) shows the layer thickness and interface width as a function of height for the different state points. The most striking feature of the observed wetting layers is their large absolute thickness $\gtrsim 1 \mu \mathrm{m}$ over heights of a few millimeters. This is very different compared to layers stabilized by van der Waals forces [2] which are negligible for our colloid-polymer mixtures; an estimation of the Hamaker constant $A$ [26] gives $|A| / k_{B} T \approx 10^{-3}$. For state points $\mathrm{S} 1$ and $\mathrm{S} 2$, we find that the interface widths are rather constant with height, ( $\sim 2-3$ particle diameters), and also do not depend much on the layer thickness, see Fig. 3. However, we observe for state point S3 that the interface widens to $\approx 1.1 \mu \mathrm{m}$ and pushes itself almost $4 \mu \mathrm{m}$ away from the wall, up to $1 \mathrm{~mm}$ above the bulkgas-liquid interface. What differentiates S3 from S1 and $\mathrm{S} 2$ is its much smaller interfacial tension which is reduced by a factor of 50 and 20, respectively. Figure 3(c) shows

(a)

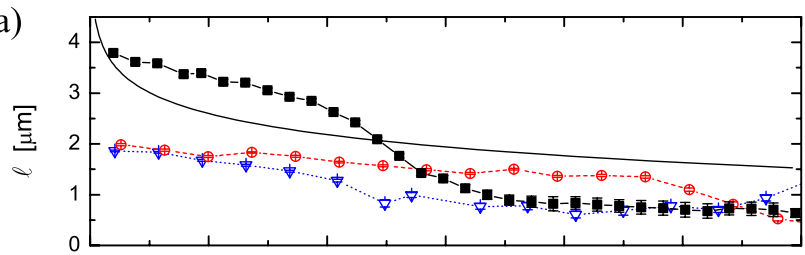

(b)

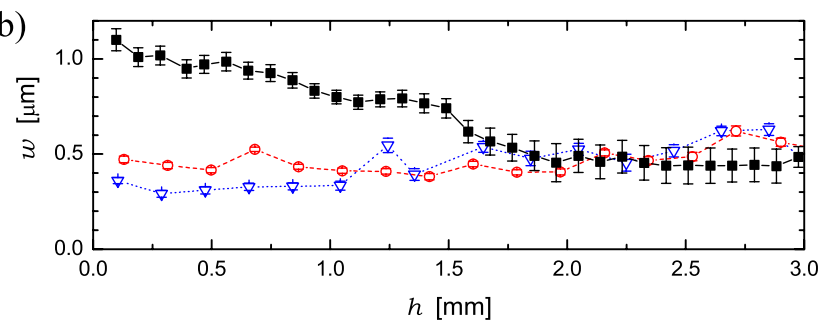

(c)

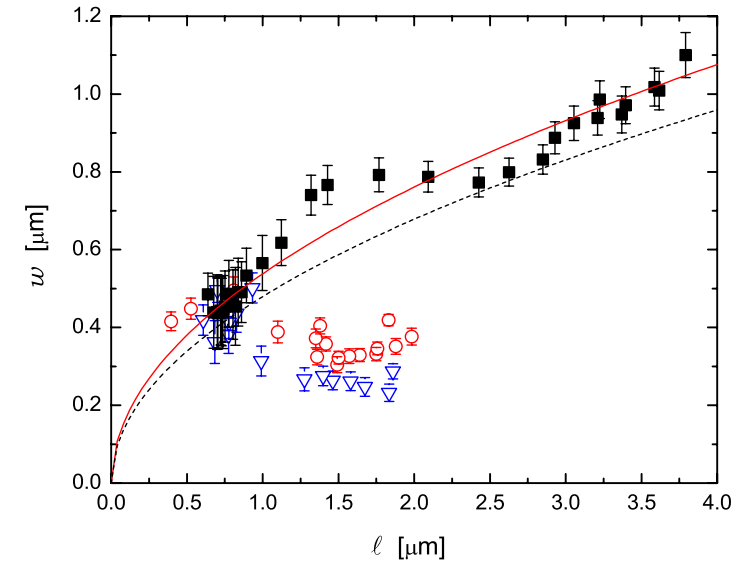

FIG. 3 (color online). Measured layer thickness $\ell$ (a) and interface width $w$ (b) as a function of height $h$ from the bulkgas-liquid interface for sample S1 (triangles), S2 (circles) and $\mathrm{S} 3$ (squares). The solid line in (a) is Eq. (7). (c) Interface width as a function of layer thickness. The solid line is $w^{2}=$ $0.29 \mu \mathrm{m} \ell$ and the dashed line is $w^{2}=0.23 \mu \mathrm{m} \ell$, describing the theoretical prediction of Eq. (3). that for this state point the roughness of the interface increases monotonically with increasing layer thickness.

Since van der Waals forces are negligible in this system, forces between surfaces decay exponentially on the scale of the (micron-sized) bulk correlation length and wetting theory for short-range forces (SRF) should apply [7,27]. Further, for the Ising universality class, the wetting parameter $\omega=k_{B} T /\left(4 \pi \gamma \xi^{2}\right)$ takes the value $\approx 0.8$ close to bulk criticality $[2,8]$. Using results of renormalization group ( $\mathrm{RG}$ ) theory for complete wetting [27,28], which incorporates thermal fluctuation effects, we derive that the mean-squared width of the confined interface depends linearly on the wetting layer thickness [29],

$$
w^{2}=f(\omega)\left(k_{B} T / \gamma\right)^{1 / 2} \ell,
$$

with $f(\omega)=(\omega / \pi)^{1 / 2} /(2+\omega)$ for $\omega<2$. The data for S3 lead to $w^{2} \approx 0.29 \mu \mathrm{m} \ell$, whereas the calculation, using $\gamma \approx 2.5 \mathrm{nN} / \mathrm{m}$ and no adjustable parameter, leads to $w^{2} \approx$ $0.23 \mu \mathrm{m} \ell$, in good agreement with the experiment.

These observations suggest that, as $\ell$ decreases, the long-wavelength modes contributing to the interface width are hindered near the wall, thus reducing $w$. The confinement of the fluctuations should therefore contribute to a fluctuation-induced pressure $\Pi_{\mathrm{fl}}(\ell)$ which pushes the interface away from the wall. According to capillary wave theory [30,31], the mean-squared displacement fluctuation $\xi_{\perp}^{2}$ of the interface is given by

$$
\xi_{\perp}^{2}=\frac{k_{B} T}{2 \pi \gamma} \ln \left(\frac{q_{\max }}{q_{\min }}\right) \equiv w^{2},
$$

where $q_{\min }=\pi / \xi_{\|}$and $q_{\max }=\pi / a$ with $\xi_{\|}$the average distance between wall-interface collisions and $a$ the particle radius. Not only collision entropy loss due to confined fluctuations, but also elastic energy cost due to increased interfacial area, contribute to the total fluctuation disjoining pressure, which is related to the associated interface potential through $\Pi_{\mathrm{fl}}(\ell) \equiv-d V_{\mathrm{fl}}(\ell) / d \ell$. Following Lipowsky and Fisher [28] we obtain

$$
\begin{gathered}
V_{\mathrm{fl}}(\ell)=\left(\gamma \xi_{\perp}^{2}+k_{B} T\right) / \xi_{\|}^{2} \\
=\frac{\left(\gamma w^{2}+k_{B} T\right)}{a^{2}} \exp \left(-\frac{4 \pi \gamma w^{2}}{k_{B} T}\right) .
\end{gathered}
$$

For state points $S 1$ and $S 2, \Pi_{\mathrm{fl}}(\ell)$ is many orders of magnitude smaller than the gravitational contribution $\Pi_{g}=\Delta \rho g h$, ruling out in these cases the possibility that the interfacial fluctuations stabilize the layer. However, for state point $\mathrm{S} 3$, at which a significant dependence of the fluctuations on the wetting layer thickness was observed experimentally, the above expression leads to a wetting layer thickness such that

$$
\left[(4 \pi-1)+\frac{4 \pi \gamma b \ell}{k_{B} T}\right] \exp \left(-\frac{4 \pi \gamma b \ell}{k_{B} T}\right)=\frac{\Delta \rho g h a^{2}}{\gamma b},
$$


where $b=0.23 \mu \mathrm{m}$ is the proportionality constant between $w^{2}$ and $\ell$. Figure 3 shows the comparison between the above theory with no adjustable parameters and our experiment. Reasonable agreement is obtained; the remaining discrepancies may indicate that the equilibrium thickness profile of the wetting layer has not been reached completely. Equilibration times for wetting layers are very long as diffusion is the only process by which equilibrium is achieved. For wetting films, this may take time on the order of several days [32]. This is typically the time we wait before doing a measurement, and we thus expect to be close to the equilibrium film thickness. In contrast, the interface fluctuations relax with a characteristic time on the order of seconds [25] and therefore adjust to the local wall thickness relatively rapidly. Further from the critical point, the large layer thicknesses observed in our experiment compared to the theoretical expectation can result from (i) direct interactions on the scale of the bulk correlation length, such as follow from the Cahn theory of wetting (see [19] for the application to colloid-polymer mixtures), (ii) residual electrostatic interactions that could lead to electrical double layer disjoining pressures - interesting charge effects have been observed recently in similar colloidal systems [33]. As demonstrated by Langmuir for water [34], surface ionization of the substrate can lead to unusually thick wetting layers of the order of $1 \mu \mathrm{m}$. However, further experiments are needed to understand the origin of the discrepancy between theory and experiment further from the critical point.

In sum, using confocal microscopy we characterized the thickness and the roughness of wetting layers in a system with an ultralow surface tension and hence large interfacial fluctuations. We find clear evidence that, close to the critical point, long-wavelength fluctuation modes are hindered in the confined fluid interface, which translates to a reduction of the interface width. Most importantly, we find good agreement with RG calculations for the exponent and amplitude of the layer thickness dependence of the roughness of the confined interface. However, the entropic repulsion seems irrelevant to the thick wetting layers found further from the critical region.

We thank S. Rafaï for helpful discussions. We also thank ICI, A. van Blaaderen and C. van Kats for providing chemicals and expertise regarding the colloid synthesis. LPS de l'ENS is UMR 8550 of the CNRS, associated with the Universities Paris 6 and Paris 7. DGALA acknowledges funding through a Marie Curie Intra-European Action, and JOI through FWO-project No. G.0483.04.

*Present address: Laboratoire Microfluidique, MEMS \& Nanostructures, ESPCI, 10 Rue Vauquelin, 75005 Paris, France.

[1] P.-G. de Gennes, Rev. Mod. Phys. 57, 827 (1985).

[2] D. Bonn and D. Ross, Rep. Prog. Phys. 64, 1085 (2001).
[3] J. W. Cahn, J. Chem. Phys. 66, 3667 (1977).

[4] M. von Smoluchowski, Ann. Phys. (Leipzig) 25, 205 (1908).

[5] M. Schick, Les Houches, Session XLVIII, Liquids at Interfaces, edited by J. Charvolin, J.-F. Joanny, and J. Zinn-Justin (Elsevier, Amsterdam, 1990).

[6] W. Helfrich, Les Houches, Session XLVIII, Liquids at Interfaces, edited by J. Charvolin, J.-F. Joanny, and J. Zinn-Justin (Elsevier, Amsterdam, 1990).

[7] E. Brézin, B. I. Halperin, and S. Leibler, Phys. Rev. Lett. 50, 1387 (1983).

[8] A. O. Parry, J. M. Romero-Enrique, and A. Lazarides, Phys. Rev. Lett. 93, 086104 (2004).

[9] K. R. Mecke and J. Krim, Phys. Rev. B 53, 2073 (1996).

[10] J. Vorberg, S. Herminghaus, and K. Mecke, Phys. Rev. Lett. 87, 196105 (2001).

[11] S. Tibus, J. Klier, and P. Leiderer, J. Low Temp. Phys. 142, 83 (2006).

[12] M. Paulus, C. Gutt, and M. Tolan, Phys. Rev. E 72, 061601 (2005).

[13] A.P. Gast, C. K. Hall, and W.B. Russel, J. Colloid Interface Sci. 96, 251 (1983).

[14] S. Asakura and F. Oosawa, J. Chem. Phys. 22, 1255 (1954).

[15] S. Asakura and F. Oosawa, J. Polym. Sci. 33, 183 (1958).

[16] A. Vrij, Pure Appl. Chem. 48, 471 (1976).

[17] J. M. Brader, R. Evans, M. Schmidt, and H. Löwen, J. Phys. Condens. Matter 14, L1 (2002).

[18] M. Dijkstra and R. van Roij, Phys. Rev. Lett. 89, 208303 (2002).

[19] D. G. A. L. Aarts, R. P. A. Dullens, D. Bonn, R. van Roij, and H. N. W. Lekkerkerker, J. Chem. Phys. 120, 1973 (2004).

[20] D. G. A. L. Aarts, J. Phys. Chem. B 109, 7407 (2005).

[21] W. K. Wijting, N.A.M. Besseling, and M.A. Cohen Stuart, Phys. Rev. Lett. 90, 196101 (2003).

[22] G. A. Vliegenthart and H. N.W. Lekkerkerker, Prog. Colloid Polym. Sci. 105, 27 (1997).

[23] E.H. A. de Hoog and H. N. W. Lekkerkerker, J. Phys. Chem. B 103, 5274 (1999).

[24] B. H. Chen, B. Payandeh, and M. Robert, Phys. Rev. E 62, 2369 (2000).

[25] D. G. A. L. Aarts, M. Schmidt, and H. N. W. Lekkerkerker, Science 304, 847 (2004).

[26] J. Israelachvili, Intermolecular \& Surface Forces (Academic Press, New York, 1992), 2nd ed.

[27] D. S. Fisher and D. A. Huse, Phys. Rev. B 32, 247 (1985).

[28] R. Lipowsky and M.E. Fisher, Phys. Rev. B 36, 2126 (1987).

[29] T. Kerle, J. Klein, and K. Binder, Phys. Rev. Lett. 77, 1318 (1996); Eur. Phys. J. B 7, 401 (1999).

[30] L. Mandelstam, Ann. Phys. (Leipzig) 41, 608 (1913).

[31] F. P. Buff, R. Lovett, and F. H. Stillinger, Phys. Rev. Lett. 15, 621 (1965).

[32] D. Bonn, E. Bertrand, J. Meunier, and R. Blossey, Phys. Rev. Lett. 84, 4661 (2000).

[33] M.E. Leunissen, C. G. Christova, A.-P. Hynninen, C.P. Royall, A. I. Campbell, A. Imhof, M. Dijkstra, R. van Roij, and A. van Blaaderen, Nature (London) 437, 235 (2005).

[34] I. Langmuir, Science 88, 430 (1938). 